\title{
Nash and Nirenberg Awarded 2015 Abel Prize
}

Editor's Note: This article was written before the sudden loss of John Forbes Nash Jr. on May 23. Nash and his wife Alicia were killed in a traffic accident in New Jersey on the final leg of their journey home from Norway where he had just received the 2015 Abel Prize. "We at the American Mathematical Society join our colleagues and friends all over the world in expressing our deep sorrow over the tragic passing of John and Alicia Nash, and we send our most heartfelt condolences to their family."-AMS President Robert Bryant.

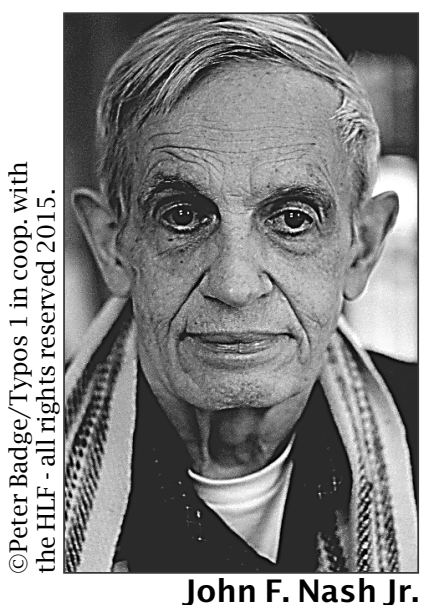

The Norwegian Academy of Science and Letters has awarded the Abel Prize for 2015 to JOHN F. NASH JR. of Princeton University and LOUIS NIRENBERG of the Courant Institute of Mathematical Sciences, New York University, "for striking and seminal contributions to the theory of nonlinear partial differential equations and its applications to geometric analysis." The Abel Prize recognizes contributions of extraordinary depth and influence to the mathematical sciences and has been awarded annually since 2003. It carries a cash award of $6,000,000$ Norwegian kroner (approximately US\$750,000). Nash and Nirenberg received the Abel Prize in an award ceremony in Oslo, Norway, on May 19, 2015.

\section{Citation}

Partial differential equations are used to describe the basic laws of phenomena in physics, chemistry, biology, and other sciences. They are also useful in the analysis of geometric objects, as demonstrated by numerous successes in the past decades.

John Nash and Louis Nirenberg have played a leading role in the development of this theory by the solution of fundamental problems and the introduction of deep ideas. Their breakthroughs have developed into versatile and robust techniques, which have become essential tools for the study of nonlinear partial differential equations. Their impact can be felt in all branches of the theory, from fundamental existence results to the qualitative study of solutions, both in smooth

DOI: http://dx.doi.org/10.1090/noti1262

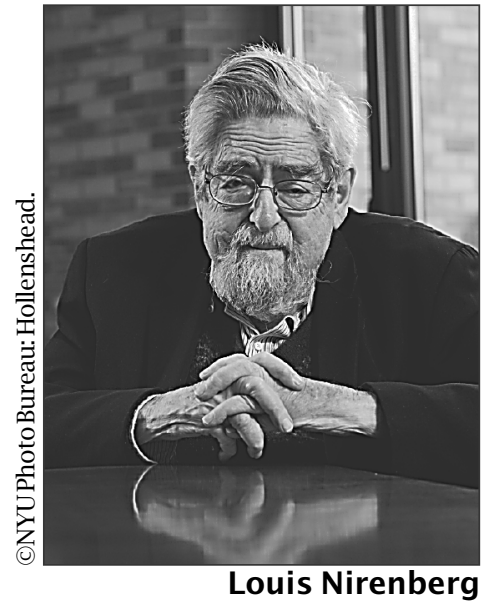

and nonsmooth settings. Their results are also of interest for the numerical analysis of partial differential equations.

Isometric embedding theorems, showing the possibility of realizing an intrinsic geometry as a submanifold of Euclidean space, have motivated some of these developments. Nash's embedding theorems stand among the most original results in geometric analysis of the twentieth century. By proving that any Riemannian geometry can be smoothly realized as a submanifold of Euclidean space, Nash's smooth $\left(C^{\infty}\right)$ theorem establishes the equivalence of Riemann's intrinsic point of view with the older extrinsic approach. Nash's nonsmooth $\left(C^{1}\right)$ embedding theorem, improved by Kuiper, shows the possibility of realizing embeddings that at first seem to be forbidden by geometric invariants such as Gauss curvature; this theorem is at the core of Gromov's whole theory of convex integration and has also inspired recent spectacular advances in the understanding of the regularity of incompressible fluid flow. Nirenberg, with his fundamental embedding theorems for the sphere $S^{2}$ in $R^{3}$, having prescribed Gauss curvature or Riemannian metric, solved the classical problems of Minkowski and Weyl (the latter being also treated, simultaneously, by Pogorelov). These solutions were important, both because the problems were representative of a developing area and because the methods created were the right ones for further applications.

Nash's work on realizing manifolds as real algebraic varieties and the Newlander-Nirenberg 
theorem on complex structures further illustrate the influence of both laureates in geometry.

Regularity issues are a daily concern in the study of partial differential equations, sometimes for the sake of rigorous proofs and sometimes for the precious qualitative insights that they provide about the solutions. It was a breakthrough in the field when Nash proved, in parallel with De Giorgi, the first Hölder estimates for solutions of linear elliptic equations in general dimensions without any regularity assumption on the coefficients; among other consequences, this provided a solution to Hilbert's nineteenth problem about the analyticity of minimizers of analytic elliptic integral functionals. A few years after Nash's proof, Nirenberg, together with Agmon and Douglis, established several innovative regularity estimates for solutions of linear elliptic equations with $L^{p}$ data, which extend the classical Schauder theory and are extremely useful in applications where such integrability conditions on the data are available. These works founded the modern theory of regularity, which has since grown immensely, with applications in analysis, geometry, and probability, even in very rough, nonsmooth situations.

Symmetry properties also provide essential information about solutions of nonlinear differential equations, both for their qualitative study and for the simplification of numerical computations. One of the most spectacular results in this area was achieved by Nirenberg in collaboration with Gidas and Ni: They showed that each positive solution to a large class of nonlinear elliptic equations will exhibit the same symmetries as those that are present in the equation itself.

Far from being confined to the solutions of the problems for which they were devised, the results proved by Nash and Nirenberg have become very useful tools and have found tremendous applications in diverse contexts. Among the most popular of these tools are the interpolation inequalities due to Nirenberg, including the Gagliardo-Nirenberg inequalities and the John-Nirenberg inequality. The latter governs how far a function of bounded mean oscillation may deviate from its average and expresses the unexpected duality of the BMO space with the Hardy space $H^{1}$. The NashDe Giorgi-Moser regularity theory and the Nash inequality (first proven by Stein) have become key tools in the study of probabilistic semigroups in all kinds of settings, from Euclidean spaces to smooth manifolds and metric spaces. The Nash-Moser inverse function theorem is a powerful method for solving perturbative nonlinear partial differential equations of all kinds. Though the widespread impact of both Nash and Nirenberg on the modern toolbox of nonlinear partial differential equations cannot be fully covered here, the Kohn-Nirenberg theory of pseudodifferential operators must also be mentioned.
Besides being towering figures as individuals in the analysis of partial differential equations, Nash and Nirenberg influenced each other through their contributions and interactions. The consequences of their fruitful dialogue, which they initiated in the 1950s at the Courant Institute of Mathematical Sciences, are felt more strongly today than ever before.

\section{Biographical Sketch: John F. Nash Jr.}

John Forbes Nash Jr. was born in 1928 in Bluefield, West Virginia. He entered the Carnegie Institute of Technology (now Carnegie Mellon University) in Pittsburgh with a full scholarship, originally studying for a major in chemical engineering before switching to chemistry and finally changing again to mathematics.

At Carnegie, Nash took an elective course in economics, which gave him the idea for his first paper, "The Bargaining Problem," which he wrote in his second term as a graduate student at Princeton University. This paper led to his interest in the new field of game theory, the mathematics of decision making. Nash's PhD thesis, "Noncooperative games," is one of the foundational texts of game theory. It introduced the concept of an equilibrium for noncooperative games, the "Nash equilibrium," which has had a great impact in economics and the social sciences. While at Princeton Nash also made his first breakthrough in pure mathematics. He described it as "a nice discovery relating to manifolds and real algebraic varieties." In essence the theorem shows that any manifold, a topological object like a surface, can be described by an algebraic variety, a geometric object defined by equations, in a much more concise way than had previously been thought possible. The result was already regarded by his peers as an important and remarkable work.

In 1951 Nash left Princeton to take an instructorship at the Massachusetts Institute of Technology. Here he became interested in the Riemann embedding problem, which asks whether it is possible to embed a manifold with specific rules about distance in some $n$-dimensional Euclidean space such that these rules are maintained.

Nash provided two theorems that proved it was true: the first when smoothness was ignored and the second in a setting that maintained smoothness. In order to prove his second embedding theorem, Nash needed to solve sets of partial differential equations that hitherto had been considered impossible to solve. He devised an iterative technique, which was then modified by Jürgen Moser, and is now known as the NashMoser theorem.

In the early 1950s Nash worked as a consultant for the RAND Corporation, a civilian think tank funded by the military in Santa Monica, California. He spent a few summers there, where his work on 
game theory found applications in United States military and diplomatic strategy.

Nash won one of the first Sloan Fellowships in 1956 and chose to take a year's sabbatical at the Institute for Advanced Study in Princeton. Basing himself in New York, he spent much of his time at Richard Courant's fledgling Institute for Applied Mathematics at New York University. There he met Nirenberg, who suggested that Nash work on a major open problem in nonlinear theory concerning inequalities associated with elliptic partial differential equations. Within a few months Nash had proved the existence of these inequalities. Unknown to him, the Italian mathematician Ennio De Giorgi had already proved this fact using a different method, and the result is known as the Nash-De Giorgi theorem.

In 1957 Nash married Alicia Larde, a physics major whom he met at MIT. In 1959 when Alicia was pregnant with their son, he began to suffer from delusions and extreme paranoia and as a result resigned from the MIT faculty. For the next three decades Nash was only able to do serious mathematical research in brief periods of lucidity. He improved gradually, and by the 1990s his mental state had recovered.

Nash has received many honors for his work. In 1994 he shared the Nobel Prize in economic sciences with John C. Harsanyi and Reinhard Selten. In 1999 he won the American Mathematical Society's Steele Prize for Seminal Contribution to Research for his 1956 embedding theorem, sharing it with Michael G. Crandall. He has been a member of the National Academy of Sciences since 1996.

\section{Biographical Sketch: Louis Nirenberg}

Louis Nirenberg was born in Hamilton, Canada, in 1925 and grew up in Montreal, where his father was a Hebrew teacher. His first interest in mathematics came from his Hebrew tutor, who introduced him to mathematical puzzles. He studied mathematics and physics at McGill University, graduating in 1945.

The summer after graduating Nirenberg worked at the National Research Council of Canada on atomic bomb research. One of the physicists there was Ernest Courant, the elder son of New York University professor Richard Courant. Nirenberg asked Ernest's wife, who was a friend of his from Montreal, to ask her father-in-law for advice about where to do graduate studies in theoretical physics. Richard Courant responded that he should study mathematics at his department at NYU.

Nirenberg began his $\mathrm{PhD}$ research under James J. Stoker, who suggested to him an open problem in geometry that had been stated by Hermann Weyl three decades previously: Can one embed isometrically a two-dimensional sphere with positive curvature into three Euclidean dimensions as a convex surface? In order to prove that one can, he reduced the problem to one about nonlinear partial differential equations. The equations in question were elliptic, a class of equations that have many applications in science.

Nirenberg's subsequent work has been largely concerned with elliptic partial differential equations, and over the following decades he developed many important theorems about them. After receiving his PhD from NYU in 1949, he stayed on as a research assistant. He was a member of the faculty of the Courant Institute of Mathematical Sciences for his entire career, becoming a full professor in 1957. Between 1970 and 1972 he was the Institute's director; he retired in 1999.

Nirenberg has written important collaborative papers with August Newlander on complex structures (1957), with Shmuel Agmon and Avron Douglis on regularity theory for elliptic equations (1959), with Fritz John introducing the function space of functions with bounded mean oscillation (1961), with David Kinderlehrer and Joel Spruck developing regularity theory for free boundary problems (1978), and with Basilis Gidas and Wei Ming Ni about the symmetries of solutions of PDEs in 1979. A paper on solutions to the Navier-Stokes equations, coauthored with Luis A. Caffarelli and Robert V. Kohn, won the American Mathematical Society's 2014 Steele Prize for Seminal Contribution to Research.

Nirenberg's awards and honors include the Bôcher Memorial Prize of the AMS (1959), the Crafoord Prize (with Vladimir Arnold, 1982), the Steele Prize for Lifetime Achievement from the AMS (1994), the National Medal of Science (1995), and the first Chern Medal for Lifetime Achievement (2010). He has been a member of the National Academy of Sciences since 1969.

\section{About the Prize}

The Niels Henrik Abel Memorial Fund was established in 2002 to award the Abel Prize for outstanding scientific work in the field of mathematics. The prize is awarded by the Norwegian Academy of Science and Letters, and the choice of Abel Laureate is based on the recommendation of the Abel Committee, which consists of five internationally recognized mathematicians.

Previous recipients of the Abel Prize are: JeanPierre Serre (2003), Michael Atiyah and I. M. Singer (2004), Peter Lax (2005), Lennart Carleson (2006), S. R. S. Varadhan (2007), John G. Thompson and Jacques Tits (2008), Mikhail L. Gromov (2009), John Tate (2010), John Milnor (2011), Endre Szemerédi (2012), Pierre Deligne (2013), and Yakov Sinai (2014).

-From an announcement of the Norwegian Academy of Science and Letters 